\title{
Nefzaoua (Nafzawa) : Moyen-âge
}

\section{Abderrahmane Khelifa}

\section{OpenEdition}

\author{
Journals
}

Édition électronique

URL : https://journals.openedition.org/encyclopedieberbere/2704

DOI : 10.4000/encyclopedieberbere.2704

ISSN : 2262-7197

\section{Éditeur}

Peeters Publishers

\section{Édition imprimée}

Date de publication : 4 octobre 2012

Pagination : 5389-5392

ISBN : 978-90-429-2640-0

ISSN : 1015-7344

\section{Référence électronique}

Abderrahmane Khelifa, « Nefzaoua (Nafzawa) : Moyen-âge », Encyclopédie berbère [En ligne], 33 | 2012, document N33, mis en ligne le 23 novembre 2020, consulté le 17 février 2022. URL : http://

journals.openedition.org/encyclopedieberbere/2704; DOI : https://doi.org/10.4000/ encyclopedieberbere.2704

Ce document a été généré automatiquement le 17 février 2022.

(c) Tous droits réservés 


\title{
Nefzaoua (Nafzawa) : Moyen-âge
}

\author{
Abderrahmane Khelifa
}

1 Dans les sources médiévales arabes, le terme désigne à la fois une ville et une tribu. La ville de Nefzaoua est décrite par El-Bekrî comme étant « située à six journées à l'ouest de Kairouan*... à trois jours de Gabès, à deux journées de Gafsa, et à une journée de Tozeur ». Elle est située en fait entre quarante cinq et cinquante lieues au sud-sud ouest de Kairouan et elle est mentionnée dans la carte de Shaw. Toujours selon El-Bekrî, « elle renferme une grande source nommée Taourgha, «jaune » en langue berbère, et dont on n'a jamais trouvé le fond. Le mur de Nefzaoua, construit en pierres et en briques est percé de six portes». Cette ville semble assez importante puisqu'elle possède une mosquée cathédrale (djama') et des bazars très fréquentés. Elle est située à proximité d'une rivière dont « les bords sont couverts de dattiers et d'autres arbres fruitiers. A proximité de la ville on trouve un grand nombre de sources».

2 El-Watwat donne une description assez semblable en affirmant que la ville, ceinte d'un rempart, est « située sur une rivière autour de laquelle s'étend une montagne. C'est la métropole du pays; elle a des faubourgs, des marchés et une banlieue plantée de palmiers dont la quantité lui donne une ressemblance avec Baçra. Il y passe trois rivières de chacune desquelles se détachent six dérivations, dont l'eau répartie à l'aide de rigoles sert à l'arrosage des vergers ".

Ibn Khaldoûn parle des villages des Nefzaoua, dans la province de Qastiliya, qui sont situés à peu de distance les uns des autres. Il affirme qu'il y avait des Francs qui vivaient dans ces villages sous la protection d'un traité encore en cours, depuis la conquête musulmane jusqu'à l'époque où vivait Ibn Khaldoûn (XIV siècle). Ibn Khaldoûn ajoute : «Comme ils professent une des croyances tolérées par l'islamisme, ils jouissent du libre exercice de leur religion et en paient la capitation ».

4 El-Idrîssî affirme que Daris est fils de Lawi ibn Nafzaou. Nafzaou étant l'éponyme de tous les Nafzaoua. Il ajoute plus loin que « de la ville de Tozeur à Nefzaoua il y a une journée et demi. Au sud de celle-ci, à cinq étapes, du côté du djebel Nefousa*, est la ville de Zarud, au sud, un peu plus de deux journées ». La tribu des Nefzaoua comporte, selon Ibn Khaldoûn, un grand nombre de branches, telles que les Oulhassa, les Ghassassa, les Zehila, les Soumata, les Ourcif, les Mernissa, les Zatima, les Ourkoul, les Ouerdeghrous, 
les Ourdin... Toutes ses familles descendent d'Itouwaft fils de Nefzaou. Ibn Idhârî ElMerrakuchî affirme que les Nafzaoua seraient une fraction des Luwata*. Nafzaou étant le fils de Luwa El-Saghîr. Il qualifie Tarîq ibn Ziyad* de Louati de Nefzaoui et d'Oulhassi quand celui-ci se trouvait à Tlemcen pour préparer l'expédition contre la péninsule Ibérique. El-Bekrî les signale dans Aoudaghost* où ils sont les plus nombreux des Berbères.

Ibn Abd al-Hakam nous apprend que dans la guerre qui opposa Handhala Ibn Safwân ElKalbî, gouverneur d'Egypte contre 'Ukâcha b. Ayyûb al-Fazârî allié de Abd El-Wahîd Ibn Yazid El-Hawwarî El-Madhami, un soufrite commandait la région de Tlemcen $(124 \mathrm{H} /$ 741-42). Cantonné dans Kairouan, Handhala réussit à battre séparément ses ennemis. Puis il écrivit à Mueâwiya b. Șafwân, son gouverneur de Tripoli, pour lui demander de réprimer une révolte de Berbères Nefzaoua. Muعâwiya fut tué mais les Nafzaoua furent vaincus.

6 Ibn Khaldoûn écrit qu'en l'an 140 H/757-58, des Nefzaoua participèrent aux côtés des Ouferdjouma à la révolte qui opposa le gouverneur de Kairouan, Abd El-Rahmân Ibn Habîb, qui avait répudié l'autorité du Khalife Abû Dja'fâr El-Mançoûr, à ses frères El-Yâs et Abd El-Ouâreth qui l'assassinèrent. Le fils du gouverneur, Habîb, tua El-Yâs mais Abd El-Ouâreth parvint à se réfugier dans les Aurès chez les Ouferdjouma où il obtint l'appui de leur émir Acem Ibn Djemîl qui fit proclamer l'autorité du Khalife El-Mançoûr. Il marcha sur Kairouan en entraînant les Nefzaoua. Cette tribu comptait en son sein des guerriers de valeur comme Abd El-Malek ibn Abi-l-Djâd et Yazîd ibn Seggoûm. Devenus maitres de Kairouan en 140 H/757-58), les Nafzaoua, qui professaient la doctrine ibâdite, massacrèrent tous les Qoreïchites et les Arabes restés dans Kairouan. Ibn Khaldoûn écrit :

«ils attachèrent leurs montures dans la grande mosquée et commirent tant d'autres forfaits et profanations qu'ils excitèrent l'indignation des Berbères ibâdites de Tripoli qui vinrent occuper Kairouan en passant au fil de l'épée les Nefzaoua et les Ouferdjouma."

7 Plus tard (161 H/777-78), les Nefzaoua se soulevèrent contre Daoud fils Yazîd et professèrent ouvertement la doctrine ibâdite. Ils avaient à ce moment comme chef Saleh ibn Noceïr. Ils livrèrent bataille à Sicca Veneria (le Kef) et furent pratiquement exterminés.

8 L'Anonyme du Kitâb El-Istibçâr décrit le territoire des Nefzaoua comme une région qui fait partie du Djérid. Ce canton est très peuplé et il s'y trouve des villes, des Ksours et de nombreux champs cultivés. Le Kitâb El-Istibçâr cite les agglomérations de T'orra, Bichchara, Aïtemlin qui sont des villes ceintes de murailles avec des palmiers, des oliviers* et des arbres fruitiers de toute sorte. Dans la ville même de Nefzaoua, il y a une source importante appelée en berbère Taourgha qui est de facture ancienne. Le Kitâb El-Istibçâr signale à proximité, une ville antique en ruine appelée El-Medina. ElBekrî et l'Anonyme du Kitâb El-Istibçâr signalent que de la ville de Nefzaoua à Qastiliya, il fallait compter une journée de marche à travers des marais salins (sebkha) qui étaient balisés de poutres au-delà desquelles les voyageurs disparaissaient sans laisser de trace.

Les luttes qu'entreprirent les Nefzaoua durant tout le Moyen âge contre les différents pouvoirs firent chuter leur démographie. Ils se divisaient en plusieurs branches qui sont: les Ghassassa (littoral algéro-marocain), les Mernissa (dans les Traras), les Sumata, les Oulhassa (autour de l'embouchure de la Tafna), les Ouardaghrous, les Ourdine, les Warkul, les Ouarsif, les Zahila, les Zatima, les Maklata, les Madjar. 
10 tribu, furent occupés par les Béni Soleïm, appartenant tous à la famille de cherid. Il y avait également des Zoghba. Ibn Khaldoûn affirme que ces Arabes possédaient les terres labourables et les fermes de ces contrées. Ces villages obéissaient au seigneur de Tozeur sous l'autorité hafside au $\mathrm{XIV}^{\mathrm{e}}$ siècle mais, aussitôt que l'autorité se relâchait, ces villages reprenaient leur indépendance

\section{BIBLIOGRAPHIE}

IBN ABD EL-HAKAM, Conquête de l'Afrique du Nord et de l'Espagne, traduction A. Gateau, Carbonel, Alger, 1947.

EL-BEKRî, Description de l'Afrique septentrionale, traduction de Slane, Paris, Geuthner, 1965.

EL-IDRÎSSî, Le Maghreb au XII siècle, (VI siècle de l'Hégire), texte établi et traduit d'après Nuzhat ElMushtaq par M. Hadj Sadok, Alger, OPU, 1983.

IBN IDHARI EL-MERRAKUCHI, Kitâb El-Bayân El-Maghreb fi akhbâr El-Andalûs wa El-Maghrib, Leiden Brill. IBN KHALDOÛN A., Histoire des Berbères et des dynasties septentrionales de l'Afrique du Nord, 4 tomes, traduction de Slane, Geuthner, Paris, 1978.

IBN KHALDOÛN Y., Histoire des Béni Abd El-Ouad, rois de Tlemcen, traduction A. Bel, Fontana, Alger, 1903.

DACHRAOUI F., Le califat fatimide au Maghreb 296-362/909-973, histoire politique et institutions, Tunis, STD, 1981

POTIRON G., Tribus (Liste des tribus berbères), Mémoire de D.E.S., Université d'Alger, 1956

\section{INDEX}

Mots-clés : Géographie, Moyen Âge, Tribu, Tunisie 